\title{
Fully Automatic 3D Threat Image Projection: Application to Densely Cluttered 3D Computed Tomography Baggage Images
}

\author{
Najla Megherbi, Toby P. Breckon, Greg T. Flitton, Andre Mouton \\ School of Engineering, Cranfield University, Bedfordshire, UK \\ e-mail: n.megherbi, toby.breckon, g.t.flitton, a.mouton@cranfield.ac.uk
}

\begin{abstract}
In this paper, we describe a Threat Image Projection (TIP) method designed for 3D Computed Tomography (CT) screening systems. The novel methodology automatically determines a valid 3D location in the passenger 3D CT baggage image into which a fictional threat 3D image can be inserted without violating the bag content. According to the scan orientation, the passenger bag content and the material of the inserted threat appropriate CT artefacts are generated using a Radon transform in order to make the insertion realistic. Densely cluttered 3D CT baggage images are used to validate our method. Experimental results confirm that our method is able to reliably insert threat items in challenging 3D images without providing any perceptible visual cue to human screeners.
\end{abstract}

Keywords - Image processing applications, Computed Tomography, Aviation Security, Threat Image Projection.

\section{INTRODUCTION}

TIP is software that is incorporated in the current X-ray baggage screening machines in order to asses human airport screener performance in the detection of threat items. For cabin baggage screening, X-ray images of realistic threat items are projected into the existing X-ray images of the passenger bags being scanned. By contrast, in hold baggage screening, TIP systems project X-ray images of whole bags containing threat items into the scanned image sequence the human operator sees. TIP is important because threat objects are difficult to recognize in images due to bag complexity, object orientation, object superposition and especially if they are irregular such as improvised explosive devices (IEDs) [3], [4]. By exposing the screeners to a considerable number of threat items during their normal screening operations, TIP has the potential to enhance their vigilance and attention and thus improve their threat detection performance. The use of TIP technology is currently limited to the conventional 2D X-ray baggage screening systems in which the screener looks at the 2D X-ray image of the bag being scanned [5], [6], [3], [4].

CT technology initially developed for 3D medical imaging applications is in increased use in airports for baggage inspection [2]. In addition to the 2D X-ray image of the scanned bag, these new 3D volumetric CT scanning systems produce cross-sectional slices of the scanned bag and thus generate volumetric views of its content. By constructing a 3D image representation of the scanned bag, 3D volumetric CT scanning systems allow the screener access to information which is not available in 2D X-ray imagery and provide better viewing and content interpretation as they can identify hidden and otherwise occluded objects in a scanned bag.

Extending the use of TIP technology in the 3D volumetric CT scanning systems would be of great importance for enhancing screener threat detection performance. Instead of superimposing 2D images of threat items into the 2D images of passenger bags, 3D TIP inserts 3D CT threat objects in the 3D CT image of the passenger bag. To fulfil this task effectively, it is necessary to insert the threat object in an appropriate place in the passenger bag without violating the existing baggage contents, for example cutting other objects. In addition, as it is well known, the use of CT technology in scanning objects creates imaging artefacts in the reconstructed 2D and 3D images [12], [13]. Unlike medical imagery where CT artefacts have several causes [12], CT artefacts in baggage imagery are mainly due to the presence of metal objects. In practice, most scanned bags contain metal (zips, buckles...). Since most threat items are made from metal, their insertion within the baggage CT scans makes the metal artefacts more complex as this increases the amount of metal in the scanned bag. In order to make the $3 \mathrm{D}$ TIP realistic, appropriate CT artefacts have to be generated in the resulting 3D TIP images.

In the literature, only one attempt has been made in [7] to integrate 3D TIP within 3D CT scanning systems. In this work the authors handle only non-cluttered trays and the threat items are manually located within these trays. In our work, we consider densely cluttered baggage scans that reflect real passenger bags. In addition, the insertion place within the bag is determined automatically. In [7], the original CT artefacts produced by the threat item from its original scan are added to the resulting 3D TIP regardless of the bag content. In practice, this is not valid as the production of CT metal artefacts depends on the scan orientation of the object, the material of the object, and the bag content. In addition, this provides perceptible visual cues to human screeners as the artefacts originating from the inserted threat item would be inconsistent with the original bag artefacts.

In our work, in order to fulfil the task of metal CT artefacts generation, a method inspired from the well established techniques of metal artefacts reduction (MAR) in medical imaging applications is used. The MAR techniques process directly in the sinogram space (also known as Radon space or projection space) [8], [9], [10]. We offer a novel approach to realistic 


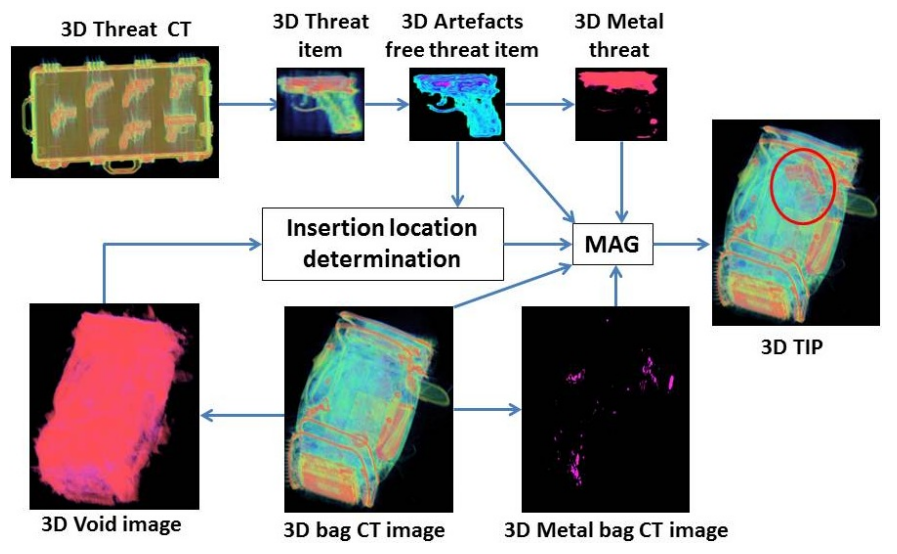

Fig. 1. The flow chart of the proposed 3D TIP method

3D TIP with adaptive metal artefacts generation that extend the prior works [3], [4], [5], [6], [7].

\section{3D THREAT IMAGE PROJECTION USING RADON TRANSFORM}

\section{A. Overview of our approach}

The architectural overview of our 3D TIP methodology is illustrated in Figure 1. Our overall approach consists of three main modules: (a) void determination; (b) threat insertion location determination; (c) Metal Artefacts Generation (MAG). Our 3D TIP approach starts first by automatically finding the empty space (void) within the scanned bag so the threat item can be inserted into the bag without intersecting other objects. Once a void is determined, an insertion location is determined automatically according to the size of the threat item. The result of this process is fed to the MAG routine along with the original CT images of the clear bag and the threat item and the spatial map of their significant metal part (obtained by thresholding). In the MAG module all images are mapped to Radon space by using the Radon transform. According to the metal traces in the sinogram of both clear bag and threat item, the sinogram of the clear bag is then modified to produce similar metal-like CT artefacts.

\section{B. Void determination}

The problem of void determination is a crucial step in 3D TIP systems. Indeed, unlike conventional X-ray systems in which the TIP insertion is realized by a simple superposition of a threat image into the $2 \mathrm{D}$ image of the passenger bag, in 3D TIP systems the void in the bag must be first determined to allow a realistic threat object insertion. For instance, the threat object must be positioned correctly in the bag without violating the existing baggage contents.

As the 3D CT reconstructed images are composed of a region corresponding to the bag and an empty region surrounding the bag, a Region of Interest (ROI) for a void search inside the bag should be first determined to prevent the 3D TIP system inserting a threat outside the bag.
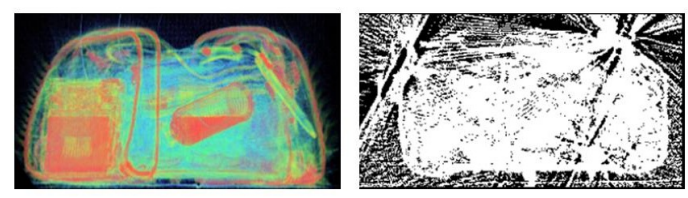

Fig. 2. Noise in CT baggage images. Thresholding the CT images reveals the considerable noise. Left: an original CT image, right: it's a binary CT image obtained using a very low threshold value

In our application, a bag ROI is generated by first isolating the bag boundary and then segmenting the outer region. The bag boundary is used to guide the segmentation process of the outer region.

In segmentation techniques it is well known that to segment a region successfully, the contour of this region should be continuous and closed. If such a condition does not hold the segmentation process will always leak outside the contours at the boundary of the region which presents the discontinuity. In practice a region boundary can be extracted using three techniques: edge detection, thresholding and active contour models (snakes)/deformable models [18]. In our application CT baggage images are corrupted by a considerable amount of noise, as depicted in Figure 2, and so the use of active contour models to extract the bag boundary is ineffective. The application of edge detectors leads to a disconnected boundary and the gaps between the boundary segments is considerable. The use of CT density image thresholding is also ineffective. If a low threshold is used, the bag outer region, which we attempt to segment, is considerably affected by noise and this dramatically affects the segmentation results as the noise reduces the capacity of the segmentation process to grow large regions. By choosing a large threshold value, the noise in the bag outer region can be removed however this leads to a discontinuous bag boundary.

After a number of experiments we have followed the steps shown in Figure 3 which have proven to be the most suitable for our application in determining a bag ROI.

As depicted in this figure thresholding using a small threshold value first takes place to make the edges of the bag boundary relatively thick which makes it harder for the segmentation process to escape through the boundaries. In order to remove noise and extract the bag boundary simultaneously, we have applied connected component analysis on the resultant binary image. All objects with fewer pixels than a given size will be discarded in this step. As the noise in the binary image is presented as a set of small blobs it will be successfully removed. Subsequently, a morphological dilation operation is applied to the biggest component which correspond to the bag and its boundary to make the bag boundary even thicker so no leakage occurs when the segmentation is conducted in the bag outer region.

In order to segment the bag outer region we use region growing segmentation techniques [1] which are the most efficient segmentation methods for our application as the bag 


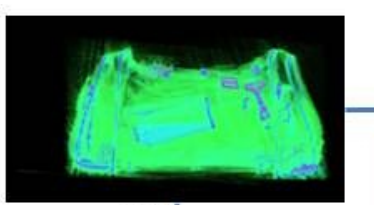

(a)

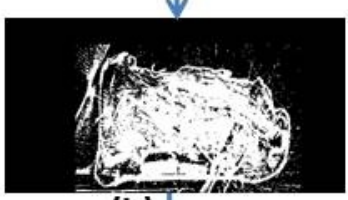

(b)

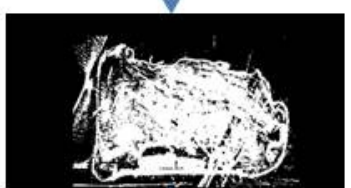

(c)

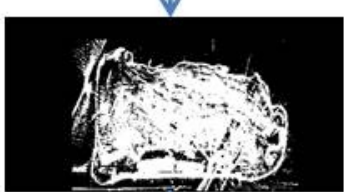

(d)

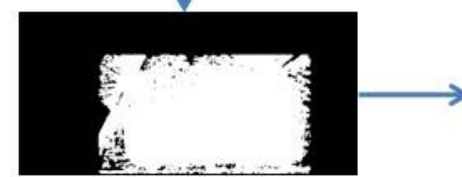

(e)

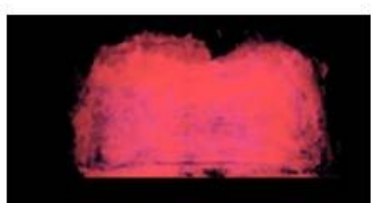

(i)

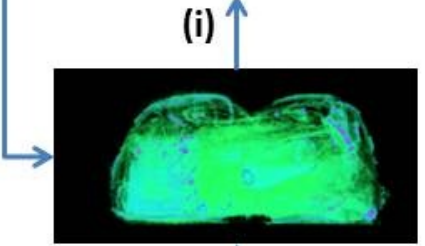

(h)

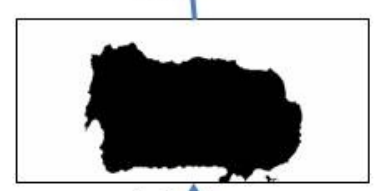

(g)

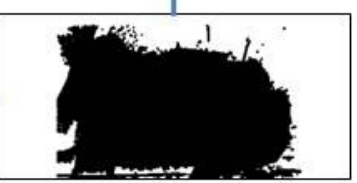

(f)

Fig. 3. 3D Void determination algorithm in CT baggage images. (a) original 3D CT image, (b) 3D binary image, (c) 3D connected components, (d) biggest component, (e) dilation, (f) initial segmented bag outer region, (g) final segmented bag outer region, (h) 3D ROI, (i) 3D bag void

outer region is made completly homogeneous by the previous steps. The seed region is set to one of the image corners as they belong to the bag outer region. This makes our process of bag outer region segmentation fully automatic.

We next apply a morphological dilation to the resulting segmented region to smooth the bag boundary. Finally to obtain the ROI, the segmented bag outer region is removed from the original CT image. The result of this process is shown in Figure 3.

Finally to determine the void from the extracted 3D ROI, we set ROI voxels having a CT density value under a given threshold to zero. This threshold is determined experimentally such that no perceptible CT baggage data lose occurs. In theory, a void in 3D images is defined as the set of voxels having zero CT density value. However, in practice this is not valid as the CT baggage images are dramatically corrupted by noise. In addition as we are considering cluttered bags, empty spaces in some cases could not be found. Thus by setting ROI voxels having low CT density value to zero, this create more empty spaces as low CT density objects such as clothes are

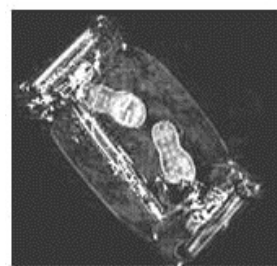

(a)

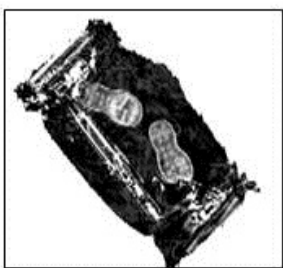

(b)

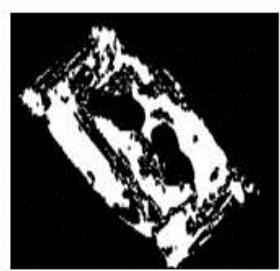

(c)
Fig. 4. Main steps of our 3D Void determination algorithm: (a) an original CT bag slice (b) its corresponding ROI, (c) void determined by our method

removed from the bag. In Figure 4 we show the main steps of this process by displaying 2D CT slices.

\section{Threat insertion location determination}

In order to find an appropriate place to insert the threat item in the void determined in the previous section, the void is divided into adjacent rectangular boxes. The size of these boxes is the same as the threat volume size. For each box a measure of insertion is computed. Currently in our work we define this measure as the ratio between the void voxels and the non-void voxels. The box having the highest insertion measure is used as the 3D space into which the threat item will be inserted. However, as in our work we are considering cluttered bags, finding an appropriate insertion place is not often possible. For this reason an insertion index threshold is used to validate the insertion. If the highest insertion measure is lower than this threshold (set to 0.5), the threshold used to find the void in the previous step is set to a lower value such that low density objects are removed to create more void in which the threat item can be inserted. This process is only repeated once and if no appropriate place still not determined, a message appears indicating that the insertion is not possible due to the bag clutter.

\section{Metal Artefacts Generation (MAG)}

1) Metal Artefacts: Metal artefacts in X-ray CT scanning are a major problem in medical imaging applications. Metal artefacts are caused by the presence of high density objects such as dental fillings and artificial hip prosthesis in the scan field of view. The origin of metal artefacts has been studied extensively in the literature and two main assumptions have been made. We refer to the first assumption as missing data assumption and to the second as nonlinearity effects assumption [14], [15], [8], [9]. In the missing data assumption, since metal objects are high-attenuation objects with a high atomic number, they heavily attenuate the X-ray beams and consequently only few photons reach the scanner detectors. This effect known as photon starvation effect produces corrupt or missed data in the measured projection data (sinogram). Consequently, during the $\mathrm{CT}$ image reconstruction process using the Filtered Back Projection (FBP) algorithm which is the standard method of CT image reconstruction, the missing data in the sinogram gets amplified by the logarithm involved 


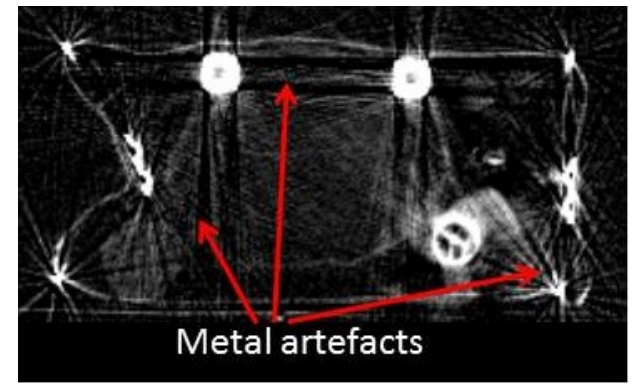

Fig. 5. Metal artefacts in a CT baggage image: Dark and bright streaks spreading across the entire CT image. Notice the strong artefacts near to the metal object.

in this algorithm and leads to artefacts in the reconstructed CT images [8], [9]. The nonlinearity effects assumption about CT metal artefacts origin states that all artefacts including metal artefacts result from the discrepancies between the actual nonlinear acquisition process and the mathematical model used in the reconstruction process. According to this assumption, CT artefacts are due to the system nonlinearities which are not included in the reconstruction model. These nonlinearities include beam hardening effect, partial volume effect, scatter effect, exponential edge-gradient effect, beam starvation effect and noise. Therefore, the mathematical imperfections of these nonlinear effects result in inconsistent data in the measured projection data [14], [15].

Regardless the origin of metal artefacts, the effects of these artefacts in the reconstructed CT images is the same. Metal artefacts appear like dark and white streaks radiating from the metal objects and spreading across the whole reconstructed CT images (see Figure 5). They are more prominent near to the metal objects. As depicted in Figure 6, metal artefacts are more visible in CT slices than the generated CT volumes (in particular for cluttered bags). They are a function of the scan orientation of the bag and the material of the bag content. In particular the material of the objects located in the shadow of the metal object affects the intensity of the metal artefacts and their direction. The high CT density these objects have the strong metal artefacts. In extreme case when several metallic objects are present, pronounced metal artefacts are produced in the reconstructed CT image [16] as depicted in Figure 6

2) Metal Artefacts Generation (MAG) for $3 D T I P$ : In our application we are processing threat items mostly made from metal. According to the above discussion, it is necessary to take into consideration metal artefacts in the TIP process in order to make the threat item appear as if it were genuinely located in the scanned bag.

Indeed, inserting a threat item directly in the CT image void determined in the previous step makes the TIP result unrealistic and implausible with reference to the existing baggage contents as the artefacts originating from the inserted threat item are not consistent with those of the original CT bag. As consequence, the TIP system fails due to the presence of

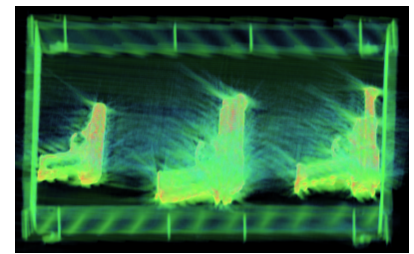

(a)

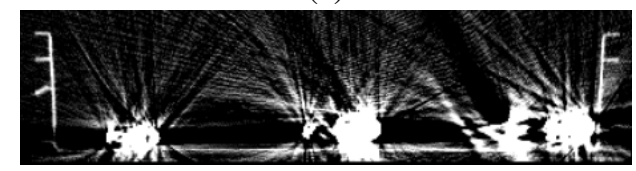

(b)

Fig. 6. Metal artefacts in the presence of several metallic objects: the intensity of the artefacts and their direction depend on the number of present metallic objects, their size and their location to each other in the bag.(a): 3D CT image, (b): a 2D CT slice

visual cues (caused by these artefacts inconsistency) that help the human screeners to identify easily a TIP image.

In Figure 7(a) we show a slice of a real scanned bag in which CT artefacts are obvious in the proximity of metal objects. In Figure $7(\mathrm{~b})$ we show the case when a gun is inserted directly in the bag image void without removing its original CT artefacts produced during its real scan. As can be seen, the TIP looks fictional as the artefacts originating from the gun are inconsistent with the bag artefacts. Figure 7(c) shows the case of inserting an artefact free gun. As can be seen, although the material of the gun is mostly metal there are no artefacts originating from the gun as opposed to the other metal objects in the same bag. This lack of artefacts around the gun makes the threat insertion looks fictional as well. As a consequence, in the following we incorporate MAG in our approach as a final step which takes into consideration the bag content and threat item materials to make the threat items appear as if they were genuinely located in the scanned bag and thus make them indistinguishable from the CT nature of the baggage environment.

Our MAG procedure depicted in Figure 8 is inspired from the MAR works in [8], [9]. Our method makes use of the clear bag (harmless bag) CT image, the artefact free threat item CT image and their CT metal-only images. The clear bag metalonly image contains all segmented metal objects existing in the bag while the threat item metal only-image contains the metal part of the threat item. Both metal-only images can be produced by either thresholding original $\mathrm{CT}$ images or thresholding original projection data. Setting a metal threshold in the CT domain is easier than in projection domain so we choose to work with virtual sinograms computed by Radon transform [17] of all the data instead of using those provided by the CT scanner. However, in order to mimic the CT scanner used during the real scans, the CT scanner geometry and the projection parameters used to compute the Radon transform are defined according to the actual CT scanner geometry and its parameters. This enables our method to include the scan 


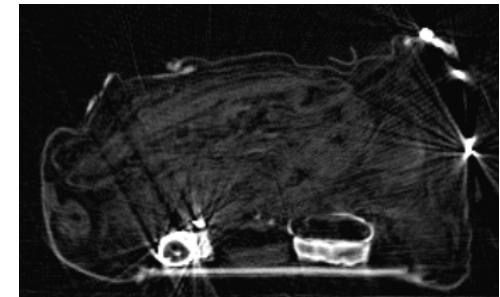

(a)

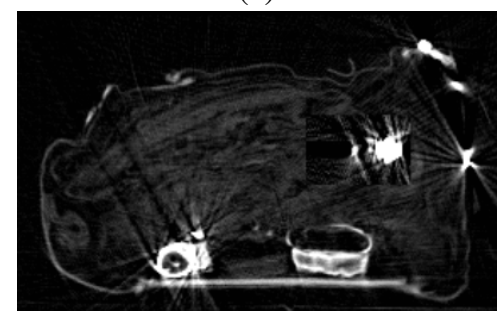

(b)

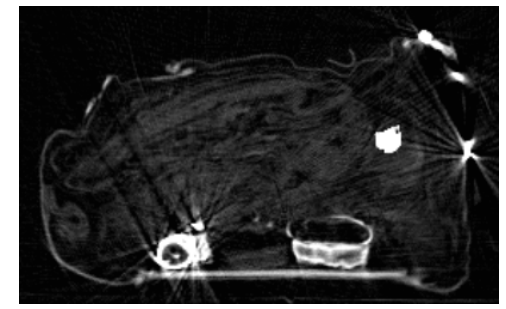

(c)

Fig. 7. (a) CT bag slice before performing TIP, (b) TIP using oiginal threat item artefacts, (c) TIP using an artefact free threat item

orientation of the bag in the MAG process. The artefact free threat item 3D CT image is obtained first by isolating the 3D threat item from its surroundings in the threat CT image and then applying a suitable CT thresholding to remove the artefacts and the noise.

Our MAG procedure starts first by mapping the original 3D CT image of the clear bag, its metal-only 3D CT image and the 3D CT metal-only image of the artefact free threat item to the projection domain via Radon transform. The output of this step is images known as sinograms. The metal traces corresponding to the sinograms of the metal-only images are subsequently combined in one projection image. A mask corresponding to the whole metal traces is then marked in the sinogram of the clear bag CT image. Marking this region in the Radon domain is equivalent to determining all lines (rays) passing through the metallic objects (clear bag metal objects and threat item metal part) in the CT domain when performing the projection. In order to generate metal artefacts in the bag CT image, the values of the bag sinogram in the masked region are modified. The underlying idea behind this is to make the sinogram values inconsistent with their neighbourhood if the corresponding rays have intersected metal objects. Indeed, since metal objects are high-attenuation objects, they heavily attenuate the $\mathrm{X}$ ray beams and consequently only few photons reach the scanner detectors. This effect known as photon starvation effect produces indeed corrupted data in the sinogram and gives rise to artefacts in the reconstructed $2 \mathrm{D}$ and $3 \mathrm{D}$ images.

In order to corrupt the projection bins of the marked mask in the clear bag sinogram, we have used an empirical function. As we will show shortly, by following the above approach, consistent metal artefacts are generated within the bag CT images. As depicted in Figure 8, once the metal artefacts are generated in the Radon space, the resulting modified sinogram is reprojected into the $\mathrm{CT}$ domain. The resulting reconstructed CT image corresponds to the original clear bag CT image corrupted by metal artefacts originating from the threat item and the bag metal objects as well. The final 3D TIP image is obtained by combining the resulting CT image with the artefact free threat item CT image.

Before moving to the following section it is important to indicate that the insertion location determined automatically by our system is also used in the Radon space in order to place the threat item metal trace in the projection data.

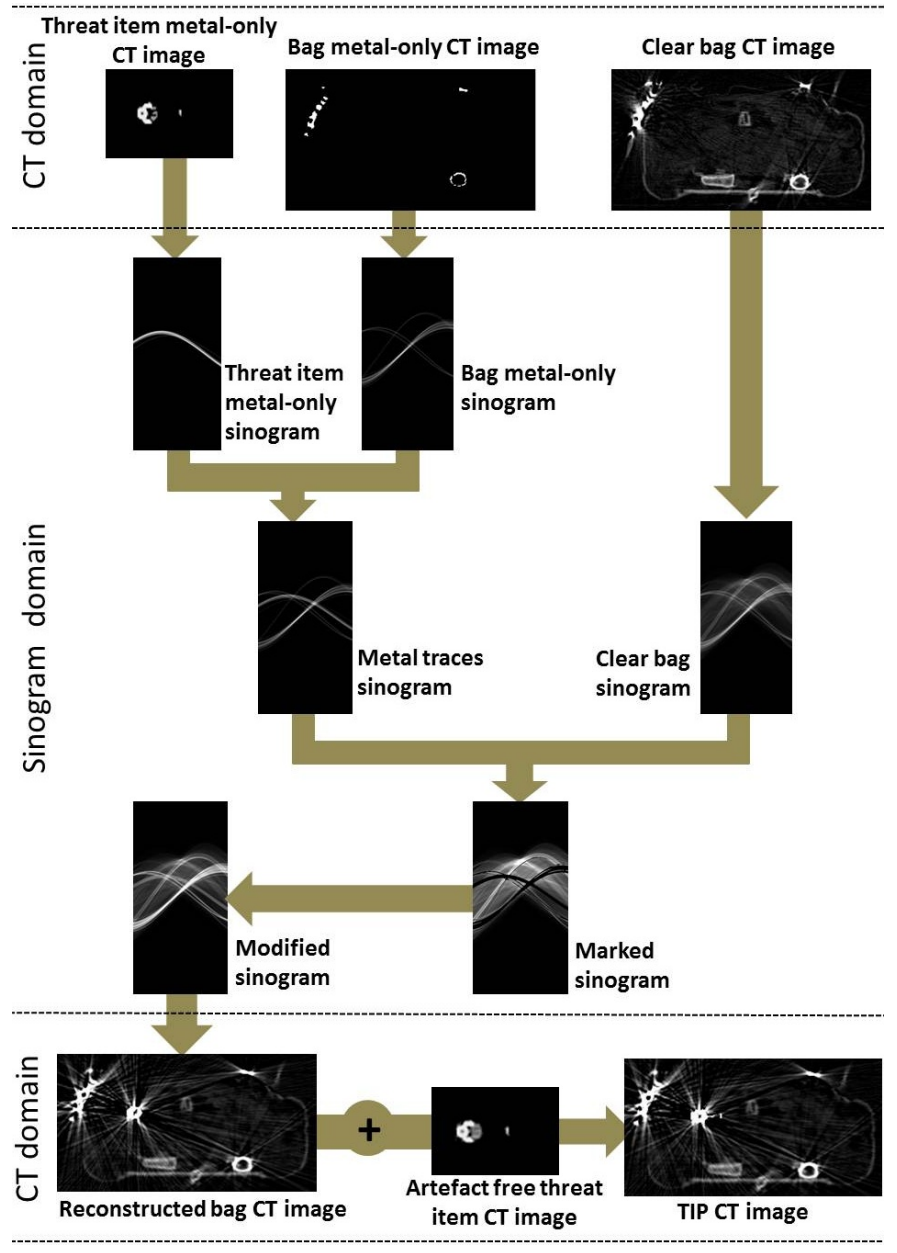

Fig. 8. Flow chart of our MAG method depicted using 2D CT slices

\section{EXPERIMENTAL RESULTS}

Our 3D TIP method is experimentally validated using challenging densely cluttered 3D CT baggage images that reflect 


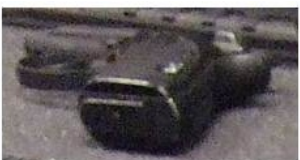

(a)

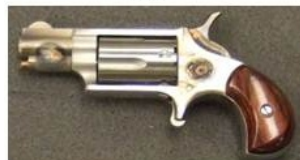

(c)

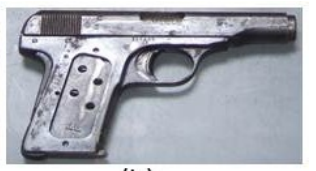

(b)

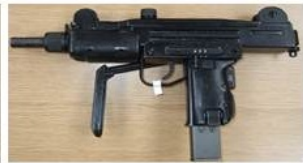

(d)
Fig. 9. Threat items used in the experiments

real passenger bags. As in our 3D TIP method we are more interested in metal artefacts we used guns as threat items as they are mostly made from metal. In Figure 9 we show four guns used for this experiment.

In Figures 10- 13, we show the results of inserting the 3D CT images of the above guns in four cluttered 3D CT baggage images using our 3D TIP method. Look crefully for the inserted items. In addition, as it is difficult to see the MAG results in 3D images, we have selected one representative slice for each case which highlights the performance of our method.

As the above examples illustrate, it is clear that the insertion of the threat items for the four cases looks realistic. The threat items are positioned appropriately inside the bag and the generated CT artefacts are consistent with those of the bag. In addition, it is clearly shown that the generated metal CT artefacts are originated from the metallic parts of the threat item and the clear bag.

In Figure 14, we show a failure case of our automatic insertion location determination process when a gun is partially inserted inside a doll. This problem occurs in particular when a clear void space is not found in the bag due either to the noise such in this example or the bag clutter. In such situations the void located inside low density and empty objects such as dolls and mugs is considered by our method as a potential place in which a threat item can be inserted. Work is in progress to exclude this void in order to have a realistic emplacement of the threat items.

Since we are using real data, a qualitative evaluation of our method is very difficult. But since TIP systems are used in passenger bags visual inspection by the aviation screeners, we have asked three experts in CT baggage data to look carefully at our 3D TIP images and evaluate the insertion performance. None of them has determined that the threat items are not parts from the scanned bags and that they have been artificially inserted inside the bags.

\section{CONCLUSION}

In order to test the aviation screeners with regards to their efficiency at determining threats, TIP is used. For this end, images of realistic threat items are projected amongst the passenger baggage being scanned. While 3D CT screening
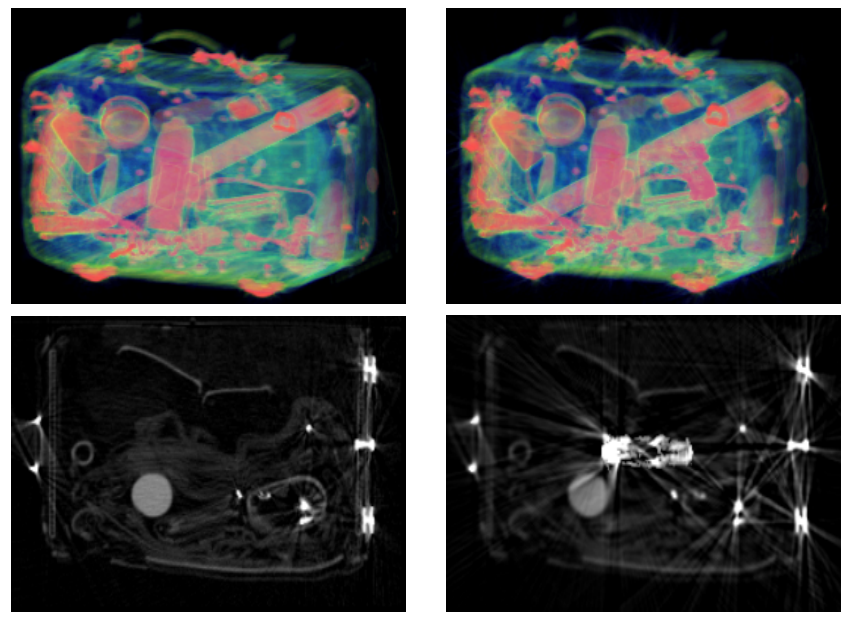

Fig. 10. Insertion of gun(a). Left: original CT images, right: TIP images. Top: 3D CT images, bottom: CT slices
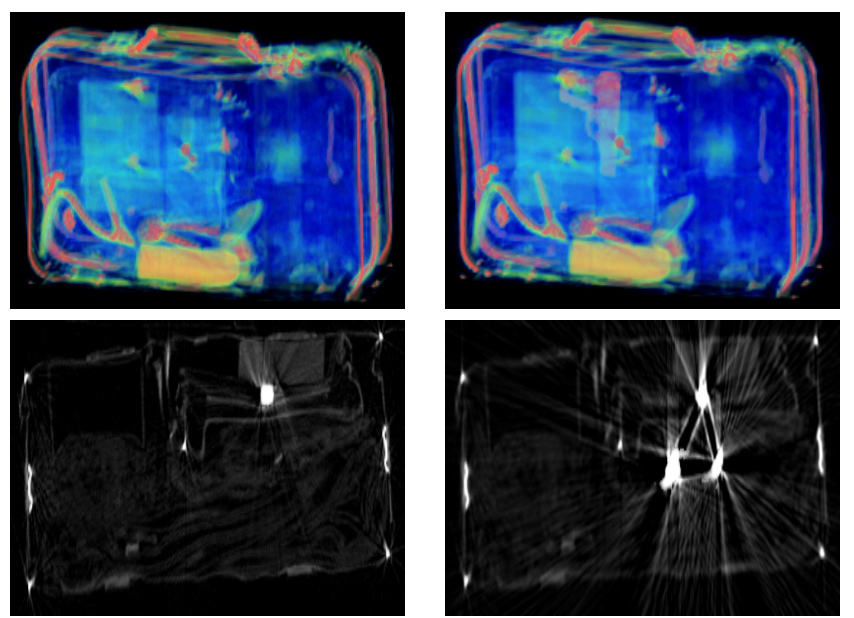

Fig. 11. Insertion of gun(b). Left: original CT images, right: TIP images. Top: 3D CT images, bottom: CT slices
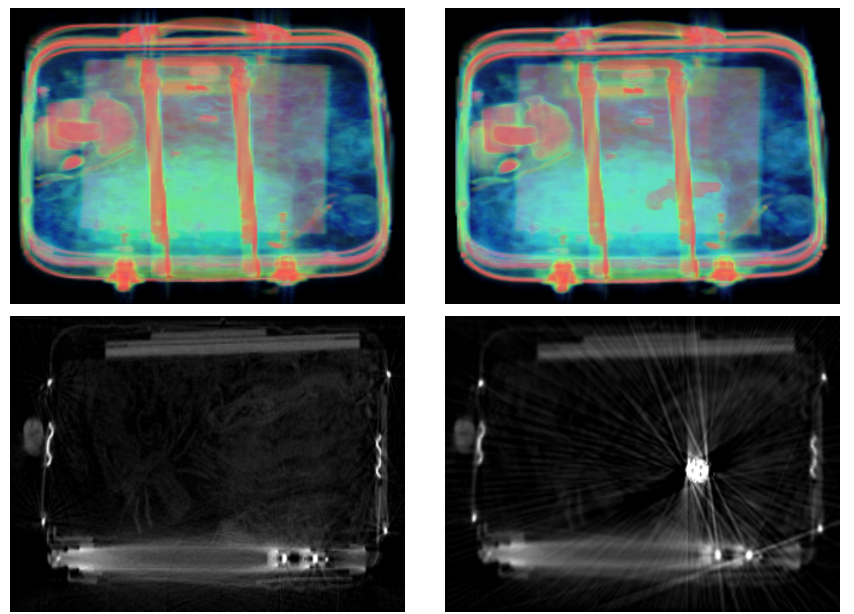

Fig. 12. Insertion of gun(c). Left: original CT images, right: TIP images. Top: 3D CT images, bottom: CT slices 
systems are widely used in airports, current TIP systems are only designed for 2D X-ray images. In this paper we proposed a fully automatic 3D TIP method which can be integrated within 3D CT screening systems and thus benefit from the advantage of using 3D scanner machines. Given a cluttered bag, our method automatically locates an appropriate location for insertion of a threat item. Depending on the bag content and the material of the threat item CT artefacts are generated consistently within the bag CT image. The MAG is performed in Radon domain. Our new method has been tested on challenging densely cluttered 3D CT baggage images. The results obtained are very promising.

Currently, our 3D TIP method is relatively slow for online operator training. Improving the speed of our method is in progress. In addition we are working on the problem of cutting low density and empty objects by considering geometric constraints and $3 \mathrm{D}$ spatial reasoning for realistic emplacement of the threat items.
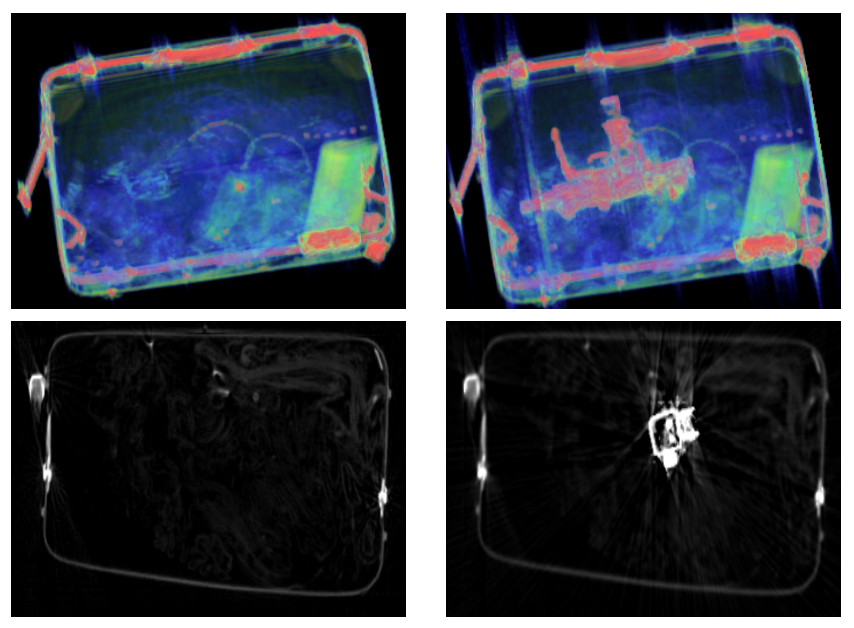

Fig. 13. Insertion of gun(d). Left: original CT images, right: TIP images Top: 3D CT images, bottom: CT slices

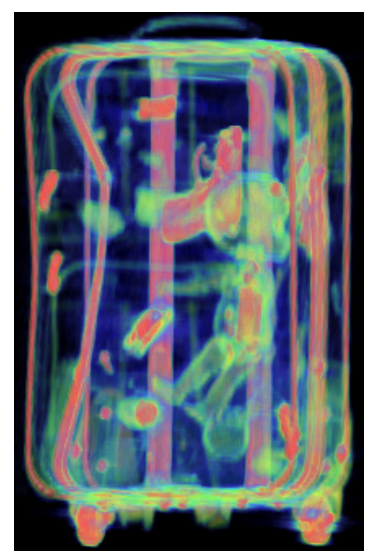

Fig. 14. Insertion failure: a gun cutting a low density object (doll)

\section{ACKNOWLEDGMENT}

This project is funded under the Innovative Research Call in Explosives and Weapons Detection (2010), a cross-government programme sponsored by Home Office Scientific Development Branch (HOSDB), Department for Transport (DfT), Centre for the Protection of National Infrastructure (CPNI) and Metropolitan Police Service (MPS).

\section{REFERENCES}

[1] R. Adams and L. Bischof. Seeded region growing. In IEEE Trans. Pattern Analysis Machine Intelligence, 16(6):641-647, 1994.

[2] S. Singh and M. Singh. Explosives detection systems (EDS) for aviation security. Signal Processing,83(1):31-55, 2003.

[3] K. Catchpole and J. Fletcher and A. McClumpha and A. Miles and A. Zar Threat image projection: applied signal detection for aviation security. Engineering Psychology and Cognitive Ergonomics,6(1):231-237, 2001.

[4] V. Cutler and S. Paddock. Use of threat image projection (TIP) to enhance security performance. 43rd Annual International Carnahan Conference in Security Technology,46-51,2009.

[5] S.M. Steiner-Koller and A. Bolfing and A. Schwaninger. Assessment of X-ray image interpretation competency of aviation security screeners. 43rd Annual International Carnahan Conference on Security Technology,20-27, 2009.

[6] F. Hofer and A. Schwaninger. Using threat image projection data for assessing individual screener performance. WIT Transactions on the Built Environment,82: 417-426, 2005.

[7] Y. O. Yildiz and D. Q. Abraham and S. Agaian and K. Panetta. 3D threat image projection Proc. SPIE ,6805, 2008.

[8] A. Mehranian and MR. Ay and A. Rahmim and H. Zaidi. Sparsity Constrained Sinogram Inpainting for Metal Artifact Reduction in X-ray Computed Tomography. IEEE Nuclear Science Symposium and Medical Imaging Conference, Barcelona, Spain, 3694-3699, 2011.

[9] A. Mehranian and MR. Ay and A. Rahmim and H. Zaidi. Metal Artifact Reduction in CT-Based Attenuation Correction of PET using Sobolev Sinogram Restoration. IEEE Nuclear Science Symposium and Medical Imaging Conference, Barcelona, Spain, 2936-2942, 2011.

[10] J. A. Kennedy and O. Israel and A. Frenkel and R. Bar-Shalom and H. Azhari. The reduction of artifacts due to metal hip implants in CTattenuation corrected PET images from hybrid PET/CT scanners. Med. Biol. Eng. Comput. ,45: 553-562,2007.

[11] C. Lemmens and D. Faul and J. Nuyts. Suppression of metal artifacts in CT using a reconstruction procedure that combines MAP and projection completion. IEEE Trans. Med. Imaging,28:250-260, 2009.

[12] J.F. Barrett and N. Keat. Artifacts in CT: Recognition and Avoidance. RadioGraphics,24, 1679-1691, 2004.

[13] M. Yazdi and L. Beaulieu. Artifacts in Spiral X-ray CT Scanners: Problems and Solutions. International Journal of Biological and Medical Sciences, 3:135-139, 2008.

[14] B. De Man and J. Nuyts and P. Dupont and G. Marchal and P. Suetens. Metal streak artifacts in X-ray computed tomography: a simulation study. IEEE Transactions on Nuclear Science, 46(3):691-696, 1999.

[15] JF. Williamson and BR. Whiting and J. Benac and RJ. Murphy and GJ Blaine and JA. O'Sullivan and DG. Politte and DL. Snyder. Prospects for quantitative computed tomography imaging in the presence of foreign metal bodies using statistical image reconstruction. Med Phys., 29(10),2404-2418, 2002.

[16] J. K. Young and Ra. J. Beom. Reduction of artifacts due to multiple metallic objects in computed tomography. Medical Imaging 2009: Physics of Medical Imaging. Edited by Samei, Ehsan; Hsieh, Jiang. Proceedings of the SPIE,7258,2009.

[17] S.R. Deans. The Radon Transform and Some of Its Applications. New York: John Wiley \& Sons, 1983.

[18] M. Kass and A. Witkin and D. Terzopoulos. Snakes: Active Contour Models. International Journal of Computer Vision,1(4):321-331, 1987. 